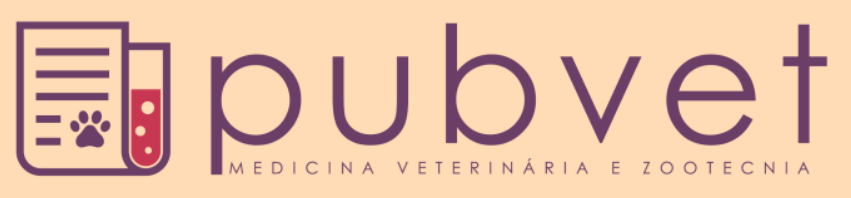

https://doi.org/10.31533/pubvet.v12n8a150.1-5

\title{
Desenvolvimento de mudas de alface em função de substratos alternativos
}

\author{
Igor Batista Morais ${ }^{1}$, Cleiton Miguel Fernandes ${ }^{1 \bullet}$, Lucas Roberto de Carvalho $^{2} \bullet$, \\ Antônio Florentino de Lima Junior ${ }^{2} \bullet$, Juracy Mendes Moreira ${ }^{2} \bullet$, Arinaldo Pereira da \\ Silva $^{2}$, Roberto Barbuio ${ }^{2}$, Juliano Queiroz Santana $\operatorname{Rosa}^{2} \odot *$
}

\author{
${ }^{1}$ Engenheiro Agronômico, \\ ${ }^{2}$ Prof. Faculdade Montes Belos. São Luiz de Montes Belos - GO. E-mail: juraci.moreira@fmb.edu.br \\ *Autor para correspondência E-mail: julianoqsr@gmail.com
}

\begin{abstract}
RESUMO. Este trabalho teve objetivo avaliar o efeito da adição de cama de frango (CF) e esterco bovino (EB) à fibra de coco (FC) na formulação de um substrato para produção de mudas de alface tipo americana. $\mathrm{O}$ experimento foi conduzido no município de Mossâmedes-GO, no período de outubro de 2016 a novembro de 2016. O cultivo foi conduzido em estufa coberta de PEBD translúcido. $\mathrm{O}$ experimento foi realizado em delineamento inteiramente casualizado, sendo sete tratamentos de substratos $(100 \% \mathrm{FC}$; $90 \% \mathrm{FC}+10 \% \mathrm{~EB} ; 80 \% \mathrm{FC}+20 \% \mathrm{~EB} ; 60 \% \mathrm{FC}+40 \% \mathrm{~EB} ; 90 \% \mathrm{FC}+10 \% \mathrm{CF} ; 80 \% \mathrm{FC}+$ $20 \% \mathrm{CF} ; 60 \% \mathrm{FC}+40 \% \mathrm{CF}$ ) e 40 repetições. Foi realizada análise física e química dos sete substratos. Avaliou-se a porcentagem de emergência; a massa do sistema radicular e parte aérea no momento do transplantio. A fibra de coco pura, como substrato, e sem fertirrigação é inviável para a produção de mudas de alface. Os substratos formulados com $80 \%$ de fibra de coco $+20 \%$ de esterco bovino curtido e $60 \%$ de fibra de coco + $40 \%$ de esterco bovino curtido são adequados para produção de mudas de Alface. A cama de frango mostrou-se uma fonte muito salina, mesmo curtida, comprometendo a emergência e desenvolvimento das mudas.
\end{abstract}

Palavras chaves: Ambiente protegido, Lactuca sativa, salinidade

\section{Development of lettuce changes in the function of alternative substrates}

ABSTRACT. This work had the objective of evaluating the effect of the addition of
chicken litter and bovine manure to the coconut fiber in the formulation of a substrate for
the production of Lucy Brown American lettuce seedlings. The experiment was
conducted in the municipality of Mossamedes-GO, from October 2016 to November
2016 . The cultivation was conducted in a greenhouse covered with translucent LDPE.
The experiment was performed in a completely randomized design, with seven treatments
of substrates (100\% FC, $90 \% \mathrm{FC}+10 \% \mathrm{~EB}, 80 \% \mathrm{FC}+20 \% \mathrm{~EB}, 60 \% \mathrm{FC}+40 \% \mathrm{~EB}$,
$90 \% \mathrm{FC}+10 \% \mathrm{CF} 80 \% \mathrm{FC}+20 \% \mathrm{CF}, 60 \% \mathrm{FC}+40 \% \mathrm{CF}$ ) and 40 replicates. Physical
and chemical analysis of seven substrates were performed. The percentage of germination
was evaluated; The mass of the root system and aerial part at the time of transplanting. It
was concluded that: pure coconut fiber without fertigation is not feasible for the
production of lettuce seedlings; The substrates formulated with $80 \%$ coconut fiber + $20 \%$
tanned bovine manure and $60 \%$ coconut fiber $+40 \%$ tanned bovine manure are suitable
for production of lettuce seedlings; The chicken bed showed to be a very saline material,
even tanned, compromising the germination and development of the seedlings.

Key words: Growing media, Lactuca sativa, salinity 


\title{
Crecimiento de mudas de lechuga en función de sustratos alternativos
}

\begin{abstract}
RESUMEN. Este trabajo tuvo el objetivo de evaluar el efecto de la adición de pollinaza (CF) y estiércol bovino (EB) a la fibra de coco (FC) en la formulación de un sustrato para producción de mudas de lechuga tipo americana. El experimento fue conducido en el municipio de Mossâmedes-GO, en el período de octubre de 2016 a noviembre de 2016. El cultivo fue conducido en invernadero cubierto de PEBD translúcido. El experimento fue realizado en delineamiento completamente casualizado, siendo siete tratamientos de sustratos $(100 \% \mathrm{FC}, 90 \% \mathrm{FC}+10 \% \mathrm{~EB}, 80 \% \mathrm{FC}+20 \% \mathrm{~EB}, 60 \% \mathrm{FC}+40 \% \mathrm{~EB}, 90 \%$ $\mathrm{FC}+10 \% \mathrm{CF}, 80 \% \mathrm{FC}+20 \% \mathrm{CF}, 60 \% \mathrm{FC}+40 \% \mathrm{CF})$ y 40 repeticiones. Se realizó análisis físico y químico de los siete sustratos. Se evaluó el porcentaje de emergencia; la masa del sistema radicular y la parte aérea en el momento del trasplante. La fibra de coco pura, como sustrato, y sin fertiirrigación, es inviable para la producción de mudas de lechuga. Los sustratos formulados con $80 \%$ de fibra de coco $+20 \%$ de estiércol bovino curtido y $60 \%$ de fibra de coco $+40 \%$ de estiércol bovino curtido son adecuados para producción de mudas de lechuga. La pollinaza se mostró una fuente muy salina, incluso curtida, comprometiendo la emergencia y el desarrollo de las mudas.
\end{abstract}

Palabras clave: Ambiente protegido, Lactuca sativa, salinidad

\section{Introdução}

A Alface é uma hortaliça folhosa bastante consumida in natura em refeições como sanduíches, saladas ou de acompanhamento a algum prato (Callegari et al. 2001). Também, possui grande importância econômica e social devido à sua adaptação a diversos climas, baixo custo de produção, fácil manejo, e ainda, por ser uma cultura pouco suscetível a pragas e doenças, a produção de mudas é uma das etapas mais importantes do sistema produtivo. Para obtenção de uma boa muda é necessário utilizar técnicas mais eficientes na escolha da semente e o uso de substratos de alta qualidade. As propriedades físicas dos substratos são importantes, tendo em vista que as relações ar e água não podem ser alteradas durante o cultivo, o que difere as características químicas, que podem ser alteradas por meio da irrigação e adubação (Costa 2013).

Na região do município de Mossâmedes-GO, a cama de frango e o esterco bovino são dois materiais facilmente encontrados e a baixo custo, tanto o esterco bovino, quanto a cama de frango são bons fornecedores de nutrientes, o que pode acarretar na redução do uso de fertilizantes minerais na produção de mudas. Segundo Sanchez (2007) a utilização de compostos orgânicos para produção de mudas proporciona um substrato com maiores teores de nutrientes, garantido melhores condições para o desenvolvimento das plantas. Contudo, apresenta baixo espaço de aeração, fazendo necessária a mistura com outros materiais como fibra de coco, casca de pinus ou vermiculita.

Dessa forma, presumisse que, o uso de cama de frango ou esterco bovino na formulação de um substrato poderia acarretar em menor custo e menor dependência de fertilizantes minerais na produção de mudas de alface. Este trabalho teve como objetivo avaliar o efeito da adição de cama de frango e esterco bovino junto a fibra de coco na formulação de um substrato alternativo para produção de mudas de alface.

\section{Material e Métodos}

O experimento foi desenvolvido no período de outubro de 2016 a novembro de $2016 \mathrm{em}$ Mossâmedes-GO (167,6'57'S; 50¹3,3'58'O, altitude de $618 \mathrm{~m})$. O experimento foi conduzido em estufa coberta de PEBD translúcido, sem controle de temperatura ou umidade, e com irrigações manuais três vezes ao dia. Foram utilizadas sementes da alface Lucy Brown, apresentando poder de germinação $95 \%$, pureza $99,9 \%$ semeadas em bandejas de poliestireno de 288 células. Como substrato utilizou-se fibra de coco pura (FC) (produto comercial Golden Mix $80^{\circledR}$ ) e misturas deste com diferentes proporções de Cama de Frango (CF) e Esterco Bovino (EB), ambos curtidos, sendo: $100 \% \mathrm{FC}$ (controle); $90 \% \mathrm{FC}+$ $10 \% \mathrm{~EB} ; 80 \% \mathrm{FC}+20 \% \mathrm{~EB} ; 60 \% \mathrm{FC}+40 \% \mathrm{~EB}$; $90 \% \mathrm{FC}+10 \% \mathrm{CF} ; 80 \% \mathrm{FC}+20 \% \mathrm{CF} ; 60 \% \mathrm{FC}+$ $40 \% \mathrm{CF}$. 
A semeadura foi realizada dia 20 de outubro de 2016. Dos dias 21 a 25 de outubro foi realizada contagem para determinação da porcentagem de emergência, considerou-se que após três dias de valores repetidos a emergência havia cessado. No dia 18 de novembro de 2016 foi finalizado o experimento com a pesagem do sistema radicular e parte aérea das mudas. Além disso, foi realizada análise física e química dos sete substratos, seguindo métodos expeditos descritos por Kämpf (2006) e discutidos por Cunha et al. (2010). Determinou-se: densidade aparente úmida, porosidade total, $\mathrm{pH} e$ condutividade elétrica.

$\mathrm{O}$ experimento foi realizado em delineamento inteiramente casualizado com sete tratamentos (substratos) e 40 parcelas (repetições), sendo cada parcela constituída de oito células da bandeja. Foi realizado teste de comparação de médias de Scoott-Knott a 5\% de significância e teste de Dunnett a 5\% de significância, adotando a $100 \%$ FC como controle.

\section{Resultados e Discussão}

Com relação às características físicas dos substratos (Tabela 1), observou-se que a densidade seca (DS) de todos os tratamentos obedeceu às recomendações de Kämpf (2006) para a utilização em bandejas, entre 100 e $300 \mathrm{~g}$ $\mathrm{L}^{-1}$. A Porosidade Total (PT), por sua vez, foi menor que $85 \%$, considerada baixa para todos os tratamentos. Este comportamento da porosidade deve ser melhor estudado, mas provavelmente é uma ineficiência do método expedito de determinação da PT para substratos a base de fibra de coco, tendo em vista que, o método descrito por Kämpf (2006) foi inicialmente descrito por Spomer (1974) para solo. Consequentemente todos os tratamentos apresentaram baixa relação poro/sólidos, que deveria ser acima de cinco.
Quanto às determinações químicas (Tabela 1), a condutividade elétrica (CE) do $80 \% \mathrm{FC}+$ $20 \% \mathrm{CF}$ e do $60 \% \mathrm{FC}+40 \% \mathrm{CF}\left(4,40 \mathrm{dS} \mathrm{m} \mathrm{m}^{-1} \mathrm{e}\right.$ $6,56 \mathrm{dS} \mathrm{m}^{-1}$, respectivamente) foi considerada alta e a da FC100\% (1,00dS m $\left.{ }^{-1}\right)$ considerada muito baixa, de acordo com Henz \& Suinaga (2009), os demais substratos apresentaram salinidade variando de $1,93 \quad \mathrm{dS} \quad \mathrm{m}^{-1}$ a $3,21 \mathrm{dS} \quad \mathrm{m}^{-1}$, consideradas adequadas pelos mesmos autores. Exceto os substratos $100 \% \mathrm{FC} ; 90 \% \mathrm{FC}+$ $10 \% \mathrm{~EB} ; \quad 80 \% \mathrm{FC}+20 \% \mathrm{~EB}$, os demais apresentaram valores de $\mathrm{pH}$ acima do intervalo ótimo (Tabela 1) de 5,4 a 6,4 como descrito por Bailey et al. (2000).

$\mathrm{Na}$ Tabela 2 verifica-se que os tratamentos $80 \% \mathrm{FC}+20 \% \mathrm{CF}$ e $60 \% \mathrm{FC}+40 \% \mathrm{CF}$ e $\mathrm{FC} 100 \%$ que apresentaram $\mathrm{CE}$ inadequados foram os que apresentaram menor porcentagem de emergência, os demais não diferiram estatisticamente pelo teste de Scott-Knott. Ao aplicar o teste de Dunnett percebeu-se também que todos os tratamentos diferem da testemunha (FC100\%), seja para melhor ou pior, colaborando com o primeiro teste. Pode-se inferir que tanto a falta de nutrientes quanto o excesso de sais comprometeram significativamente a emergência.

A redução da germinação no tratamento $100 \%$ FC também pode ser explicada pela reduzida superfície de contato. A maior granulometria da fibra de coco e seu alto espaço de aeração que reduzem a disponibilidade de água para as sementes, consequentemente dificultando embebição e desenvolvimento inicial da plântula. Segundo Kerbauy (2008) a água é o principal fator para o início da germinação, considerando-se que o embrião não cresce a menos que haja uma entrada de água nos tecidos, suficiente para gerar pressão de turgescência necessária para a expansão celular.

Tabela 1. Análise física e química dos substratos para produção de mudas de Alface Lucy Brown. Mossâmedes-GO, 2016.

\begin{tabular}{lcccccc}
\hline Substrato & DU $\left(\mathrm{g} \mathrm{L}^{-1}\right)$ & $\mathrm{DS}\left(\mathrm{g} \mathrm{L}^{-1}\right)$ & $\mathrm{PT}(\%)$ & $\mathrm{P} / \mathrm{S}$ & $\left.\mathrm{CE}(\mathrm{dS} \mathrm{m})^{-1}\right)$ & $\mathrm{pH}$ \\
\hline $100 \% \mathrm{FC}$ & 258 & 120 & 72 & 1,9 & 1,00 & 6,23 \\
$90 \% \mathrm{FC}+10 \% \mathrm{~EB}$ & 310 & 170 & 63 & 1,7 & 1,93 & 6,43 \\
$80 \% \mathrm{FC}+20 \% \mathrm{~EB}$ & 349 & 191 & 60 & 1,5 & 2,91 & 6,66 \\
$60 \% \mathrm{FC}+40 \% \mathrm{~EB}$ & 367 & 220 & 60 & 1,5 & 3,33 & 6,90 \\
$90 \% \mathrm{FC}+10 \% \mathrm{CF}$ & 302 & 166 & 62 & 1,6 & 3,21 & 6,93 \\
$80 \% \mathrm{FC}+20 \% \mathrm{CF}$ & 346 & 205 & 61 & 1,5 & 4,40 & 7,10 \\
$60 \% \mathrm{FC}+40 \% \mathrm{CF}$ & 365 & 215 & 60 & 1,5 & 6,56 & 7,50 \\
\hline
\end{tabular}

Legenda: FC = Fibra de coco; EB = Esterco bovino; $\mathrm{CF}$ = Cama de Frango; DU = Densidade úmida; DS = Densidade seca; $\mathrm{PT}=$ Porosidade Total; $\mathrm{P} / \mathrm{S}=$ Relação Poro/Sólido; $\mathrm{CE}=$ Condutividade Elétrica. Obs.: DU, DS e PT foram determinadas por métodos expeditos, segundo Kämpf (2006). CE e pH foi determinado pelo método do lixiviado (Pour Thru), segundo $\underline{\text { Cavins et al. (2000) e Kämpf (2006). }}$ 
Tabela 2. Emergência, massa da raiz e massa da parte aérea; e Relação parte aérea/Raiz de mudas de Alface Lucy Brown. Mossâmedes-GO, 2016.

\begin{tabular}{lcccc}
\hline Substrato & $\begin{array}{c}\text { Emergência } \\
(\%)\end{array}$ & $\begin{array}{c}\text { Massa raiz } \\
\left(\mathrm{g} \mathrm{planta}^{-1}\right)\end{array}$ & $\begin{array}{c}\text { Massa aérea } \\
\left(\mathrm{g} \mathrm{planta}^{-1}\right)\end{array}$ & Aérea/Raiz \\
\hline $100 \% \mathrm{FC}$ & $80,00 \mathrm{~b}$ & $0,196 \mathrm{~d}$ & $2,456 \mathrm{~b}$ & $15,1 \mathrm{a}$ \\
$90 \% \mathrm{FC}+10 \% \mathrm{~EB}$ & $96,87 \mathrm{a}$ & $0,207 \mathrm{~d}$ & $0,616 \mathrm{e}$ & $2,9 \mathrm{~d}$ \\
$80 \% \mathrm{FC}+20 \% \mathrm{~EB}$ & $93,12 \mathrm{a}$ & $0,434 \mathrm{a}$ & $1,109 \mathrm{~d}$ & $2,6 \mathrm{~d}$ \\
$60 \% \mathrm{FC}+40 \% \mathrm{~EB}$ & $5,62 \mathrm{a}$ & $0,471 \mathrm{a}$ & $1,187 \mathrm{~d}$ & $2,6 \mathrm{~d}$ \\
$90 \% \mathrm{FC}+10 \% \mathrm{CF}$ & $98,10 \mathrm{a}$ & $0,243 \mathrm{~d}$ & $1,680 \mathrm{c}$ & $7,1 \mathrm{c}$ \\
$80 \% \mathrm{FC}+20 \% \mathrm{CF}$ & $47,50 \mathrm{c}$ & $0,389 \mathrm{~b}$ & $2,735 \mathrm{~b}$ & $7,4 \mathrm{c}$ \\
$60 \% \mathrm{FC}+40 \% \mathrm{CF}$ & $44,37 \mathrm{c}$ & $0,311 \mathrm{c}$ & $3,161 \mathrm{a}$ & $80,8 \mathrm{~b}$ \\
\hline $\mathrm{CV}(\%)$ & 17,41 & 36,13 & 61,98 & 3,22 \\
\hline DMS (Dunnett) & $8 \%$ & 0,0677 & 0,6615 & 80,71 \\
\hline
\end{tabular}

Médias seguidas por mesma letra na coluna não diferem pelo teste de Scott-Knott a 5\%. Médias com barras alinhadas na coluna não diferem pelo teste de Dunnett a $5 \%$.

Observou-se que os tratamentos $80 \% \mathrm{FC}+$ $20 \%$ EB e $60 \% \mathrm{FC}+40 \%$ EB apresentaram melhor emergência (93,12\% e $95,62 \%$, respectivamente), melhor desenvolvimento do sistema radicular $(0,434$ e 0,471 , respectivamente) e melhor relação parte área/raiz (2,6 e 2,6 respectivamente) (Tabela 2). Os tratamentos com cama de frango, sobretudo, $80 \% \mathrm{FC}+20 \% \mathrm{CF}$ e $60 \% \mathrm{FC}+$ $40 \% \mathrm{CF}$, além de apresentarem menor porcentagem de emergência tiveram uma relação parte aérea/raiz muita alta indicando mudas estioladas e de sistema radicular pouco desenvolvido, isto ocorreu certamente pelo maior teor de nutrientes presentes na cama de frango indicados pelos altos teores $\mathrm{pH}$ e CE (Tabela 1).

$\mathrm{Na}$ Figura 1 é possível observar o desenvolvimento das mudas do tratamento $60 \% \mathrm{FC}+40 \% \mathrm{~EB}$, que promoveu melhor agregação do torrão. Esta característica é desejável para o produtor, pois garante qualidade da muda e menor perda nos transplantio.

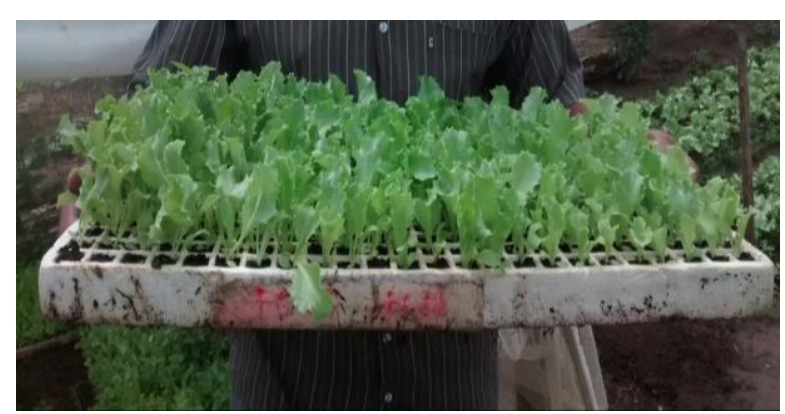

Figura 1. Bandeja com mudas do tratamento $60 \% \mathrm{FC}+40 \% \mathrm{~EB}$

Na figura 2 observa-se o tratamento FC100\%, que assim como os tratamentos com cama de frango apresentaram mudas com torrão de pior conformação quando comparados aos $80 \% \mathrm{FC} \mathrm{+}$ $20 \%$ EB e $60 \% \mathrm{FC}+40 \% \mathrm{~EB}$. Estes resultados demonstram que o esterco bovino curtido pode ser utilizado na formulação de substratos alternativos constituindo de $20 \%$ a $40 \%$ da mistura com fibra de coco, reduzindo o custo de produção das mudas, reduzindo a dependência de adubação complementar no viveiro e melhorando a qualidade das mudas. Por sua vez, a cama de frango demonstrou-se um material muito salino, mesmo curtido, seu uso na produção de mudas de alface deve ser evitado. Os dados foram analisados com ouso do software estatístico $\underline{\mathrm{R}}$ Core-Team (2016).

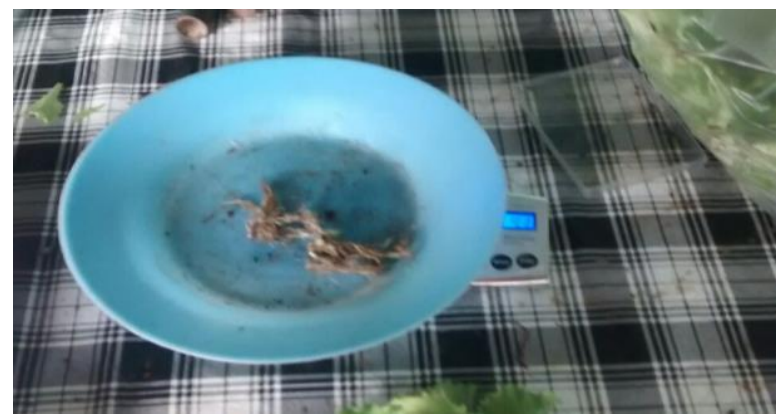

Figura 2. Muda do tratamento $100 \%$ FC.

\section{Conclusão}

O uso de Fibra de coco pura, sem adição de adubos orgânicos ou fertirrigação, é inviável para a produção de mudas de alface. Os substratos formulados com $80 \%$ de fibra de coco $+20 \%$ de esterco bovino curtido e $60 \%$ de fibra de coco + $40 \%$ de esterco bovino curtido são adequados para produção de mudas de Alface, com desempenho superior ao substrato comercial testado. A cama de frango apresentou alta salinidade, mesmo curtido. Seu uso na produção de mudas de alface compromete a germinação e provoca estiolamento das mudas, na maioria das doses avaliadas. 


\section{Referências Bibliográficas.}

Bailey, D. A., Nelson, P. V. \& Fonteno, W. C. 2000. Substrate $\mathrm{pH}$ and water quality. North Carolina State University State Extension Publications. Disponível em:<http://www.ces.ncsu.edu/depts/hort/floric ulture/plugs/ph.pdf $>$. Acesso em: 28 Nov. 2016.

Callegari O., Santos H.S. \& Scapim C.A. 2001. Variações do ambiente e de práticas culturais na formação de mudas e na produtividade da alface (Lactuca sativa L. cv. Elisa). Acta Scientiarum Agronomy 23, 1117-1122.

Cavins T.J., Gibson J.L., Whipker B.E. \& Fonteno W.C. 2000. pH and ec meters-tool for substrate analysis. North Carolina State University 1, 1-4.

Costa J.P. 2013. Desempenho de mudas de alface (Lactuca sativa) sob diferentes doses de composto orgânico. pp. 1-22, Catolé do Rocha, Paraíba.

Cunha, A. M. Cunha, G. M., Sarmento, R. A., Cunha, G. M. \& do Amaral, J. F. T. 2010. Efeito de diferentes substratos sobre o desenvolvimento de mudas de Alface sp. Revista Árvore, 30(2), 207-214.
Henz G.P. \& Suinaga F.A. 2009. Tipos de alface cultivados no Brasil. In: INFOTECA-E (ed. by EMBRAPA), pp. 1-7, Brasília.

Kämpf A.N. 2006. Floricultura: Técnicas de preparo de substratos. Editora LK., Brasília, DF.

Kerbauy G.B. 2008. Fisiologia Vegetal. Guanabara Koogan, Rio de Janeiro, Brasil.

R-Core-Team 2016. R: A language and environment for statistical computing. R. Foundation for Satatical Computing, Viena.

Sanchez S.V. 2007. Avaliação de cultivares de alface crespa produzidas em hidroponia tipo NFT em dois ambientes protegidos em Ribeirão Preto (SP).

Spomer L.A. 1974. Two classroom exercises demonstrating the pattern of container soil water distribution. HortSci 9, 1-4.

Recebido: 18 Mai. 2018.

Aprovado: 4 Jun. 2018

Publicado: 30 Jul. 2018

Licenciamento: Este artigo é publicado na modalidade Acesso Aberto sob a licença Creative Commons Atribuição 4.0 (CC-BY 4.0), a qual permite uso irrestrito, distribuição, reprodução em qualquer meio, desde que o autor e a fonte sejam devidamente creditados. 